\title{
Dynamics of viscous grounding lines
}

\author{
ROSALYN A. V. ROBISON, HERBERT E. HUPPERT \\ AND M. GRAE WORSTER $†$ \\ Institute of Theoretical Geophysics, Department of Applied Mathematics and Theoretical Physics, \\ CMS, Wilberforce Road, Cambridge CB3 OWA, UK
}

(Received 19 September 2008; revised 28 October 2009; accepted 29 October 2009)

We have used viscous fluids in simple laboratory experiments to explore the dynamics of grounding lines between marine ice sheets and the freely floating ice shelves into which they develop. We model the ice sheets as shear-dominated gravity currents, and the ice shelves as extensional gravity currents having zero shear to leading order. We consider the flow of viscous fluid down an inclined plane into a dense inviscid 'ocean'. A fixed flux of fluid is supplied at the top of the plane, which is at 'sea level'. The fluid forms a gravity current flowing down and attached to the plane for some distance before detaching to form a freely floating extensional current. We have derived a mathematical model of the flow that incorporates a new dynamic boundary condition for the position of the grounding line, where the gravity current loses contact with the solid base. The grounding line initially advances and eventually reaches a steady position. Good agreement between our theoretical predictions and experimental measurements and observations gives confidence in the fundamental assumptions of our model.

\section{Introduction}

Global warming is predicted to cause a rise in sea level of a few tens of centimetres over the next 100 years mostly in consequence of thermal expansion of the oceans and also as a result of melting inland glaciers and ice sheets (IPCC report 2007). However, the potential for much greater increases in sea level resides in the massive ice sheets of Greenland and Antarctica. Continental ice sheets, those with an inland terminus, are controlled by a balance between snowfall at high elevation and melting downstream. In contrast, melting plays a minor role in the mass balance of many marine ice sheets, which terminate in the ocean and exhibit a balance between snowfall inland and calving of icebergs into the ocean. The ice sheets of West Antarctica, for example, sit on bed rock that is one to two kilometres below sea level. They thin as they flow towards the ocean and eventually detach from the bed rock to form freely floating ice shelves. The locus of detachment is called the grounding line.

The ice sheets spread slowly under their own weight and, on long length scales and over long periods of time, flow in a manner that can be successfully modelled using continuum fluid dynamics. Typically, ice sheets are modelled using a shallowice approximation and Glen's flow law (Paterson 1994): in other words, as gravity currents with non-Newtonian (power law) rheology. While in contact with bed rock, 
the flow of an ice sheet is resisted by shear stresses, both internal and at its base, where it can be lubricated by melt water and/or glacial till (water-saturated silt and clay). However, beyond the grounding line, the ice shelf, as the sheet has become, is characterized by a weak internal straining flow, with negligible tangential stress exerted upon it by the air above it or the ocean beneath.

The rheology of the fluid forming the sheet and shelf primarily alters the vertical velocity profiles within them but is of secondary importance to the depth-averaged quantities we are interested in here. Therefore, for clarity of exposition and for ease of experimental quantification, we confine this study to Newtonian fluids.

The leading-order shallow-ice equations have a different character in shelf and sheet, and there is a significant challenge in matching them across the grounding line, which is a free boundary whose evolution must also be determined. For example, Weertman (1974) argued on the basis of perfectly plastic ice that the ice thickness should be approximately continuous across the grounding line and that the tensile stress should also balance. Most recently, Schoof (2007) has used a condition of continuity of the depth-averaged longitudinal stress across the grounding line in a non-Newtonian, viscous shallow-ice model to calculate steady solutions and determine their asymptotic characteristics in certain limits. Alternatively, Wilchinsky \& Chugunov (2000) had suggested that the shelf should leave the grounding line tangentially to the bed. The local flow in the transition region around the grounding line has been explored numerically by Nowicki \& Wingham (2008), who solved a two-dimensional freeboundary problem using the Stokes equations. Their results suggested that perhaps the second derivative of the thickness of the shelf is zero at the grounding line but they were not able to obtain a unique steady solution unless the sheet was allowed to slip at its base.

There is as yet no consensus on how to model grounding lines appropriately and no basis on which to assess the alternative hypotheses that have been proposed. To this end, we have designed and performed some simple laboratory experiments, using Newtonian fluids, that encapsulate the fundamental fluid-mechanical properties of flow across a grounding line between a shear-dominated sheet and a straindominated shelf. We have concurrently developed a Newtonian theory of sheet-shelf evolution through a grounding line and compared the predictions of this theory with our experimental results.

Many previous theoretical and numerical studies investigated steady states, and Vieli \& Payne (2005) suggested that there had been no reliable model of groundingline migration. Schoof's model (Schoof 2007) is implicitly time-dependent, while our model makes the time-dependent evolution of the grounding line explicit, in the manner of a Stefan condition for the free (moving) boundary between the sheet and shelf. It can therefore be used straightforwardly to explore the dynamical stability of grounding lines (cf. Wilchinsky 2001).

The setting for this paper is illustrated by figure 1 and supplementary movie 1 available at journals.cambridge.org/flm, taken from one of our experiments, which show a sheet of viscous fluid (golden syrup) flowing down a rigid slope into a dense ocean of aqueous potassium carbonate solution. At some position along the slope (the grounding line), the syrup floats off to form a flowing shelf. The grounding line migrates along the slope as the experiment proceeds but eventually reaches a fixed position. Our aim is to characterize this evolution and to predict it quantitatively.

In $\S 2$ we present our theoretical development, deriving in particular a dynamical equation for the evolution of the grounding line. The system of governing equations and boundary conditions form a type of Stefan problem, which we solve for a 


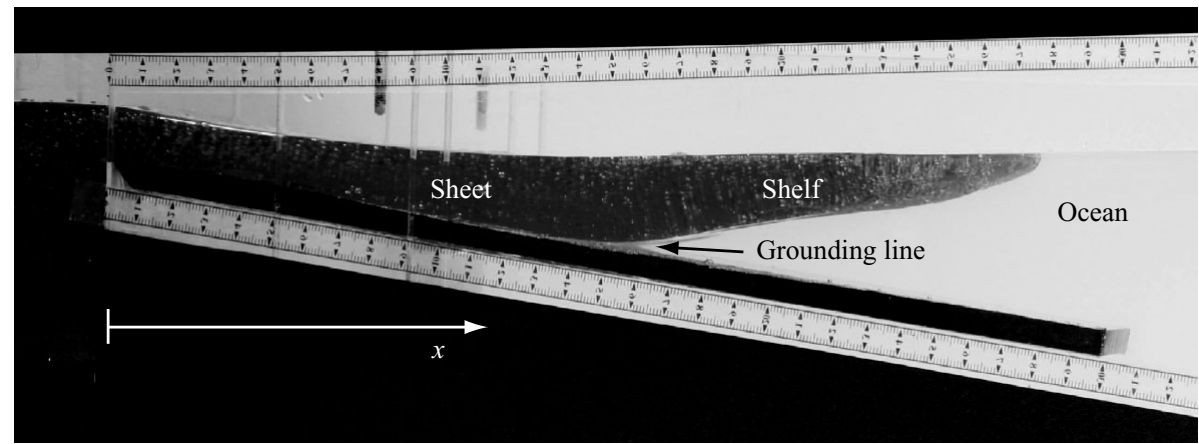

FiguRE 1. A photograph of one of our experiments. Syrup is supplied at a constant rate into a reservoir at the left, which overflows onto an inclined plane to form a sheet. Some way down the slope, at the grounding line, the sheet detaches to form a shelf floating freely on a dense ocean of potassium carbonate solution.

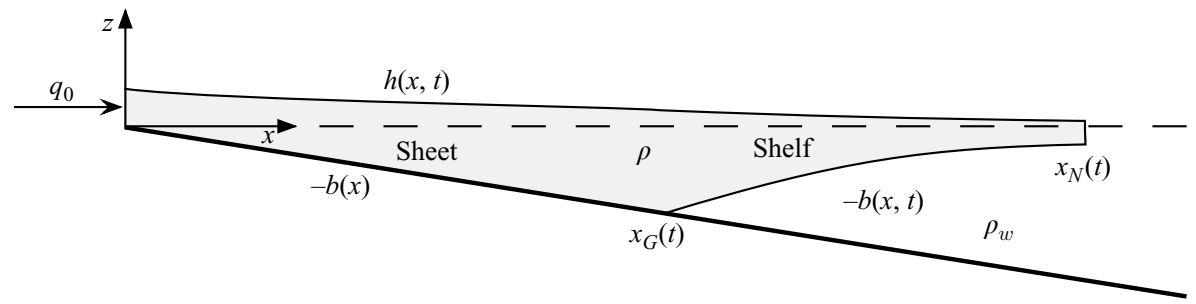

Figure 2. Schematic diagram of the flow of viscous fluid down a plane at $z=-b(x)=-\alpha x$. Beyond the grounding line, the viscous fluid forming the shelf occupies the region $-b(x, t)<z<h(x, t)$.

few special cases in $\S 3$. The experimental set-up is detailed in $\S 4$, and our results are described in $\S 5$. The experimental results and their relation to the theoretical predictions are discussed in $\S 6$, where we illustrate the roles played by sidewall stresses induced by the finite lateral extent of our experimental tank and by the rise in 'sea level' caused by the introduction of additional fluid to the tank. Our findings are summarized in the concluding $\S 7$.

\section{Theoretical development}

In this section, we develop a theory for a Newtonian viscous gravity current flowing down a slope as a sheet into a denser inviscid fluid to form a freely floating shelf. The geometry to be considered is illustrated in figure 2. Fluid of dynamic viscosity $\mu$ and density $\rho$ is introduced at the top of a rigid slope $z=-b(x, t)$, where $z=0$ (sea level) is the position of the interface between an ambient inviscid fluid of density $\rho_{w}$ and the air of negligible density above it, and $x$ measures the horizontal distance from the top of the slope. Figure 2 shows the specific example to be studied in detail in $\S 3$, in which $b=\alpha x$, for constant bed slope $\alpha$.

The fluid that is introduced forms a grounded, shear-dominated sheet, with an upper free surface at $y=h(x, t)$ and a total thickness $H(x, t)=h(x, t)+b(x, t)$ in the region $0 \leqslant x<x_{G}(t)$, where $x_{G}(t)$ denotes the position of the grounding line. In $x>x_{G}(t)$ the flow is assumed to form a freely floating shelf with an upper free surface at $z=h(x, t)$ and a total thickness $H(x, t)=\left[\rho_{w} /\left(\rho_{w}-\rho\right)\right] h(x, t)$ determined 
by Archimedes principle. The assumptions inherent in the thin-film approximation are invalid in the vicinity of the grounding line, but we relate properties of the sheet and shelf across it and seek to confirm experimentally the predictions thus made. Put another way, although there is a lot of structure to the flow in the vicinity of the grounding line, the region of adjustment between sheet and shelf is modelled here as an infinitesimal interface, which is consistent with the thin-film analysis employed.

We present the analysis in three sections: the shelf, whose evolution is determined entirely by the history of conditions at the grounding line; the sheet, which is modelled as a viscous gravity current with a free boundary at $x=x_{G}(t)$; and the grounding line, whose evolution is governed by dynamical balances across it.

\subsection{The shelf}

The freely floating viscous gravity current (shelf) in $x_{G}(t)<x<x_{N}(t)$ is assumed to be in local hydrostatic equilibrium so that

$$
\rho H=\rho_{w}(H-h) \quad \text { or } \quad h=H\left(\rho_{w}-\rho\right) / \rho_{w} .
$$

We assume that the fluids (air above and water below) bounding the shelf exert no tangential stress on it. Therefore, to leading order in lubrication theory, the horizontal velocity $u(x, t)$ is independent of depth, and has superimposed on it a pure straining flow with principal axes along the direction of flow (horizontal stretching) and perpendicular to the direction of flow (vertical thinning).

The balance of vertical forces on an element of fluid in the shelf gives

$$
\frac{\partial \sigma_{z z}}{\partial z}=\rho g
$$

where $\sigma_{i j}$ are the components of the stress tensor. Equation (2.2) is readily integrated to give

$$
\sigma_{z z}=-p_{0}-\rho g(h-z),
$$

where $p_{0}$ is atmospheric pressure, applied at $z=h$. Henceforward, we set $p_{0}=0$. Given that, for a Newtonian fluid, $\sigma_{z z} \equiv-p+2 \mu \partial w / \partial z,(2.3)$ shows that the pressure

$$
p=\rho g(h-z)+2 \mu \frac{\partial w}{\partial z}
$$

has a contribution from viscous compression as well as the usual hydrostatic contribution that dominates thin-film flows with shear. The local continuity equation $\nabla \cdot \boldsymbol{u}=0$ allows us to rewrite the pressure as

$$
p=\rho g(h-z)-2 \mu \frac{\partial u}{\partial x} .
$$

Therefore, the horizontal normal stress (longitudinal stress)

$$
\sigma_{x x} \equiv-p+2 \mu \frac{\partial u}{\partial x}=-\rho g(h-z)+4 \mu \frac{\partial u}{\partial x} .
$$

The depth-integrated longitudinal stress gives the horizontal force per unit width on a vertical cross-section of the flow as

$$
N \equiv \int_{-b}^{h} \sigma_{x x} \mathrm{~d} z=-\frac{1}{2} \rho g H^{2}+4 \mu H \frac{\partial u}{\partial x},
$$

where $b=H-h=H \rho / \rho_{w}$ is the depth of the shelf below sea level. 


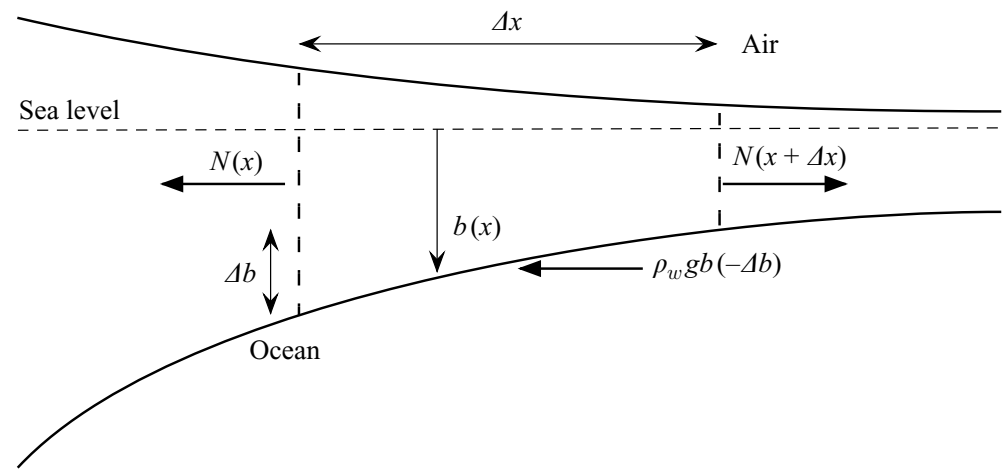

FIGURE 3. The horizontal forces on a section of shelf. The difference in depth-integrated longitudinal stress is balanced by the hydrostatic pressure from the underlying ocean, so $N(x+\Delta x)-N(x)-\rho_{w} g b(x)(-\Delta b)=0$. Equation (2.8) is obtained by dividing this equation by $\Delta x$ and taking the limit as $\Delta x \rightarrow 0$.

The overall horizontal force balance on a section of shelf fluid is described by

$$
\frac{\partial N}{\partial x}+\rho_{w} g b \frac{\partial b}{\partial x}=0
$$

(see figure 3), which can be rearranged to give the extensional flow equation

$$
\frac{4 \mu}{H} \frac{\partial}{\partial x}\left(H \frac{\partial u}{\partial x}\right)=\rho g \frac{\partial h}{\partial x}=\rho g^{\prime} \frac{\partial H}{\partial x},
$$

where the reduced gravity $g^{\prime}=g\left(\rho_{w}-\rho\right) / \rho_{w}$. This equation (or equivalently (2.8)) is readily integrated to give

$$
\frac{\partial u}{\partial x}=\frac{g^{\prime}}{8 v} H
$$

where $v=\mu / \rho$ is the kinematic viscosity of the current and in which the constant of integration has been chosen so that the vertically integrated longitudinal stress at the end of the shelf balances the integrated hydrostatic pressure in the water column adjacent to it. This latter condition is expressed by

$$
N=-\frac{1}{2} \rho_{w} g b^{2}, \quad\left(x=x_{N}(t)\right),
$$

where $x_{N}(t)$ is the position of the end of the shelf.

Equation (2.10) can be further integrated to give

$$
u(x, t)=\frac{q_{G}(t)}{H_{G}(t)}+\frac{g^{\prime}}{8 v} \int_{x_{G}(t)}^{x} H(\xi, t) \mathrm{d} \xi,
$$

where $q_{G}(t)$ and $H_{G}(t)$ are respectively the volume flux arriving at the grounding line and the thickness of the shelf at the grounding line. The integral in (2.12) is simply the volume of the shelf between the grounding line and the horizontal location $x$, and can be rewritten as a time integral of the volume flux $q_{G}^{*}(t)=q_{G}-H_{G} \dot{x}_{G}$ of fluid crossing the grounding line into the shelf. Therefore

$$
u\left(t, t_{G}\right)=\left.\frac{\partial x}{\partial t}\right|_{t_{G}}=\frac{q_{G}(t)}{H_{G}(t)}+\frac{g^{\prime}}{8 v} \int_{t_{G}}^{t} q_{G}^{*}(\tau) \mathrm{d} \tau,
$$


where we have switched to a Lagrangian description of the flow with $u\left(t, t_{G}\right)$ representing the velocity at time $t$ of the fluid at location $x\left(t, t_{G}\right)$ that left the grounding line at time $t_{G}$. A final integration of (2.13) gives

$$
x\left(t, t_{G}\right)=x_{G}\left(t_{G}\right)+\int_{t_{G}}^{t} \frac{q_{G}(\tau)}{H_{G}(\tau)} \mathrm{d} \tau+\frac{g^{\prime}}{8 v} \int_{t_{G}}^{t} \int_{t_{G}}^{\tau} q_{G}^{*}\left(\tau^{\prime}\right) \mathrm{d} \tau^{\prime} \mathrm{d} \tau .
$$

The double integral on the right-hand side of (2.14) can be integrated once, having switched the order of integration, to give

$$
x\left(t, t_{G}\right)=x_{G}\left(t_{G}\right)+\int_{t_{G}}^{t} \frac{q_{G}(\tau)}{H_{G}(\tau)} \mathrm{d} \tau+\frac{g^{\prime}}{8 v} \int_{t_{G}}^{t}(t-\tau) q_{G}^{*}(\tau) \mathrm{d} \tau .
$$

The thickness of the shelf is determined from the equation of mass conservation

$$
\frac{\partial H}{\partial t}+\frac{\partial}{\partial x}(H u)=0
$$

which can be written in Lagrangian form as

$$
\left.\frac{\partial H}{\partial t}\right|_{t_{G}}=\frac{\partial H}{\partial t}+u \frac{\partial H}{\partial x}=-H \frac{\partial u}{\partial x} .
$$

The right-hand side of (2.17) can be evaluated using (2.10), and the resulting equation integrated to yield

$$
\frac{1}{H\left(t, t_{G}\right)}-\frac{1}{H_{G}\left(t_{G}\right)}=\frac{g^{\prime}}{8 v}\left(t-t_{G}\right)
$$

Equations (2.15) and (2.18) describe the thickness of the shelf $H(x, t)$ parametrically, with parameter $t_{G}$.

As a simple concrete example, if the grounding line has a fixed position $x=x_{0}$, at which it is supplied by a constant flux $q_{G}^{*}=q_{G}=q_{0}$ and has a constant thickness $H_{G}=H_{0}$ then

$$
\begin{gathered}
x=x_{0}+\frac{q_{0}}{H_{0}}\left(t-t_{0}\right)+\frac{g^{\prime} q_{0}}{16 v}\left(t-t_{0}\right)^{2}, \\
\frac{1}{H}-\frac{1}{H_{0}}=\frac{g^{\prime}}{8 v}\left(t-t_{0}\right)
\end{gathered}
$$

from which $H(x, t)$ can be determined as

$$
H=H_{0}\left[1+g^{\prime} H_{0}^{2}\left(x-x_{0}\right) / 4 v q_{0}\right]^{-1 / 2} .
$$

Note that, in this example, $H(x, t)$ is independent of time and simply occupies the envelope described by (2.21) in the range

$$
x_{0} \leqslant x \leqslant x_{N}(t)=\frac{q_{0}}{H_{0}} t+\frac{g^{\prime} q_{0}}{16 \nu} t^{2},
$$

where $x_{N}(t)$ has been determined from (2.19) with $t_{0}=0$. The asymptotic solution at large times and large values of $x$, viz $H \sim\left(g^{\prime} x / v q_{0}\right)^{-1 / 2}$ in $0 \leqslant x \leqslant x_{N}(t) \sim\left(g^{\prime} q_{0} / 16 v\right) t^{2}$, is a similarity solution of (2.9) and (2.16), which can be determined from a scaling analysis. It shows, in conjunction with (2.21), that the shelf loses knowledge of conditions at the grounding line, other than the flux of fluid across it, at a distance $v q_{0} / g^{\prime} H_{0}^{2}$ from the grounding line.

Note from (2.15) and (2.18) that the structure and extent of the shelf are known entirely in terms of the time history of the thickness of the current at the grounding 
line $H_{G}(t)$ and the volume flux $q_{G}(t)$. Note also that the volume flux across the grounding line only determines the evolution of the shelf and does not determine the evolution of the grounding line (Schoof 2007), provided that a shelf exists (see the discussion around (2.35) below).

\subsection{The sheet}

For later use, we formulate equations here for the general case in which the base of the sheet is at position $z=-b(x, t)$. The grounded sheet, which we assume to satisfy no slip at its base, forms a classical viscous gravity current (Huppert 1982, 2000) with a parabolic velocity profile and a depth-integrated volume flux

$$
q=-\frac{g H^{3}}{3 v} \frac{\partial h}{\partial x}
$$

This expression can be substituted into the continuity equation

$$
\frac{\partial H}{\partial t}+\frac{\partial q}{\partial x}=0
$$

to form the nonlinear diffusion equation

$$
\frac{\partial h}{\partial t}=\frac{g}{3 v} \frac{\partial}{\partial x}\left[(h+b)^{3} \frac{\partial h}{\partial x}\right]-\frac{\partial b}{\partial t}
$$

for the thickness of the sheet. This is subject to the boundary conditions

$$
\begin{gathered}
-\left.\frac{g(h+b)^{3}}{3 v} \frac{\partial h}{\partial x}\right|_{x=0}=q_{0}, \\
h\left(x_{G}, t\right)=\frac{\rho_{w}-\rho}{\rho} b\left(x_{G}, t\right) .
\end{gathered}
$$

The first of these conditions reflects the imposed constant volume flux $q=q_{0}$ at $x=0$. The second condition reflects the flotation condition (2.1), which is first attained (as $x$ increases) at $x=x_{G}$.

Mathematically, the two boundary conditions $(2.26 a, b)$ would be sufficient for the second-order diffusion equation (2.25) on a fixed domain. However, a further, independent condition is required in order to determine the position of the free boundary $x=x_{G}(t)$.

\subsection{The grounding line}

An assumption incorporated into the modelling of viscous ice sheets and shelves by Schoof (2007) is that the depth-integrated longitudinal stress should balance on the two sides of the grounding line. This reflects the arguments put forward by Weertman (1974) for continuity of tensile stress in a perfectly plastic model. A numerical calculation of the fully two-dimensional flow in the neighbourhood of a Newtonian, viscous grounding line has been made by Nowicki \& Wingham (2008), and used to determine local characteristics of the flow. However, no conclusion was made relating to conditions that should apply to a depth-integrated model such as the shallow-ice model or the lubrication theory developed here. We follow the principle adopted by Schoof (2007) and derive a condition based on the continuity of longitudinal stress. 
In general, from (2.6), the depth-integrated longitudinal stress is

$$
\begin{aligned}
N & \equiv \int_{-b}^{h} \sigma_{x x} \mathrm{~d} z \\
& =-\frac{1}{2} \rho g H^{2}+4 \mu \int_{-b}^{h} \frac{\partial u}{\partial x} \mathrm{~d} z, \\
& =-\frac{1}{2} \rho g H^{2}+4 \mu\left[\frac{\partial q}{\partial x}-u(h) \frac{\partial h}{\partial x}-u(-b) \frac{\partial b}{\partial x}\right] .
\end{aligned}
$$

In the sheet, in which there is a leading-order parabolic shear flow

$$
u=-\frac{g}{2 v} \frac{\partial h}{\partial x}(z+b)[2 H-(z+b)]
$$

the depth-integrated longitudinal stress is

$$
N=-\frac{1}{2} \rho g H^{2}+4 \mu\left[\frac{\partial q}{\partial x}+\frac{g}{2 v} H^{2}\left(\frac{\partial h}{\partial x}\right)^{2}\right] .
$$

In the shelf, in which there is uniform flow to leading order, the longitudinal stress is given by (2.7). Therefore, with the simple hypothesis that $H$ and $N$ are both continuous across the grounding line,

$$
\left[\frac{\partial q}{\partial x}+\frac{g}{2 v} H^{2}\left(\frac{\partial h}{\partial x}\right)^{2}\right]_{\text {sheet }}=\left[H \frac{\partial u}{\partial x}\right]_{\text {shelf }} .
$$

Strictly, the left-hand side of (2.30) applies a sufficient distance upstream of the grounding line and the right-hand side applies a sufficient distance downstream of the grounding line for lubrication theory to apply in sheet and shelf respectively. There can therefore, in principle, be jumps in $H$ and $N$ across the interface between sheet and shelf, which in reality is an extended region but is treated here as a singular surface.

The right-hand side of (2.30) can be evaluated as $g^{\prime} H^{2} / 8 v$ using (2.10). To evaluate the left-hand side, we differentiate the flotation condition $(2.26 b)$ with respect to time, noting that $x_{G}=x_{G}(t)$, which gives

$$
\dot{x}_{G} \frac{\partial h}{\partial x}+\frac{\partial h}{\partial t}=\frac{\rho_{w}-\rho}{\rho}\left(\dot{x}_{G} \frac{\partial b}{\partial x}+\frac{\partial b}{\partial t}\right), \quad\left(x=x_{G}(t)\right) .
$$

This can be rearranged using the continuity equation to give

$$
\left(\frac{\rho_{w}-\rho}{\rho} \frac{\partial b}{\partial x}-\frac{\partial h}{\partial x}\right) \dot{x}_{G}+\frac{\rho_{w}}{\rho} \frac{\partial b}{\partial t}=\frac{\partial H}{\partial t}=-\frac{\partial q}{\partial x}, \quad\left(x=x_{G}(t)\right) .
$$

Finally we combine this with (2.30) to obtain,

$$
\left(\frac{\rho_{w}-\rho}{\rho} \frac{\partial b}{\partial x}-\frac{\partial h}{\partial x}\right) \dot{x}_{G}=\frac{g}{2 v} H^{2}\left(\frac{\partial h}{\partial x}\right)^{2}-\frac{g^{\prime}}{8 v} H^{2}-\frac{\rho_{w}}{\rho} \frac{\partial b}{\partial t}, \quad\left(x=x_{G}(t)\right) .
$$

This is a general equation governing the one-dimentional propagation of a Newtonian, viscous grounding line when the flow from the sheet crosses it into a shelf. For the 
particular fixed geometry shown in figure 2 , we can rewrite it as

$$
\left(\alpha \frac{\rho_{w}}{\rho} \frac{g^{\prime}}{g}-\frac{\partial h}{\partial x}\right) \dot{x}_{G}=\frac{g^{\prime}}{8 v} H^{2}\left[\frac{4 g}{g^{\prime}}\left(\frac{\partial h}{\partial x}\right)^{2}-1\right], \quad\left(x=x_{G}(t)\right) .
$$

If the surface slope of the sheet at the grounding line is large (for example, at early times in the example studied in $\S 3)$ then (2.34) predicts that $H \dot{x}_{G} \sim-\left(g H^{3} / 2 v\right) \partial h / \partial x$, which is greater than the flux of fluid $q=-\left(g H^{3} / 3 v\right) \partial h / \partial x$ that can be supplied by the sheet. In that case, the fluid from the sheet simply adds to the end of the grounded sheet, without forming a shelf, and the front of the sheet is determined kinematically by

$$
\dot{x}_{G}=\frac{q_{G}}{H_{G}}=-\frac{g H^{2}}{3 v} \frac{\partial h}{\partial x}, \quad\left(x=x_{G}(t)\right)
$$

because the ocean provides insufficient normal stress to balance that in the sheet. This is the propagating front condition for a spreading viscous gravity current.

Equations (2.25), (2.26) and (2.34) or (2.35) together form a type of Stefan problem. The explicit evolution equation (2.34) will allow dynamical questions, for example relating to stability of grounding lines, to be addressed straightforwardly.

\section{Some theoretical results}

The full system of equations governing the viscous sheet illustrated in figure 2 comprises (2.25), (2.26), (2.33) and (2.35). We consider the particular case in which $b(x, t)=\alpha x$ is independent of time and make these equations dimensionless by writing

$$
h=B C \hat{h}, \quad x=2 B C^{4} \hat{x}, \quad q_{0} t=2 B^{2} C^{5} \hat{t},
$$

where $B=\left(6 v q_{0} / g\right)^{1 / 3}$ and $C=\left(g / g^{\prime}\right)^{1 / 6}$. This gives, dropping hats,

$$
\begin{gathered}
\frac{\partial h}{\partial t}=\frac{\partial}{\partial x}\left[(h+A x)^{3} \frac{\partial h}{\partial x}\right], \\
h^{3} \frac{\partial h}{\partial x}=-1 \quad(x=0), \\
h=\epsilon x_{G} \quad\left(x=x_{G}\right), \\
\dot{x}_{G}=\min \left\{\begin{array}{l}
\frac{3}{2}(h+A x)^{2}\left[\left(\frac{\partial h}{\partial x}\right)^{2}-1\right] /\left(\epsilon-\frac{\partial h}{\partial x}\right) \\
-(h+A x)^{2} \frac{\partial h}{\partial x}
\end{array}\right\} \quad\left(x=x_{G}\right),
\end{gathered}
$$

where

$$
A=2 \alpha\left(\frac{g^{\prime}}{g}\right)^{-1 / 2} \quad \text { and } \quad \epsilon=2 \alpha\left(\frac{g^{\prime}}{g}\right)^{1 / 2} \frac{\rho_{w}}{\rho} .
$$

Note that the bed slope $\alpha$ should be small for lubrication theory to be valid, and that $g^{\prime} / g$ is also small. Therefore $\epsilon$ is typically a small parameter, which can safely be set to zero in the model. The dimensionless bed slope $A$ is also small in most natural settings, but for generality we allow it to be order unity throughout our discussions and make no further approximations. Given the particular geometry in this section, $A$ must be strictly positive in order for there to be any ocean. However, $A$ can be made arbitrarily small if it is desired to study that limit. 
(a)

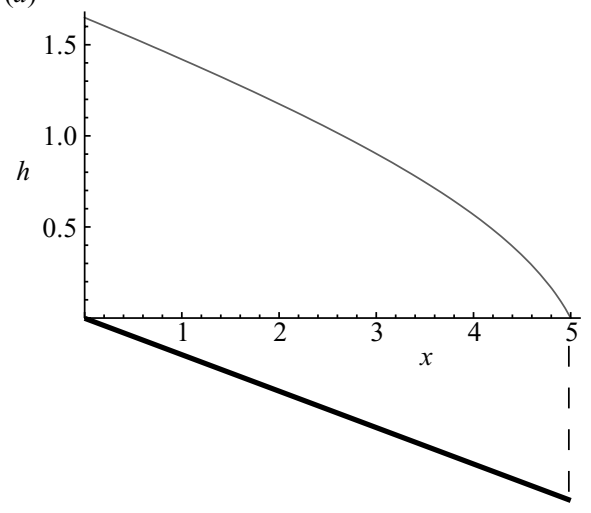

(b)

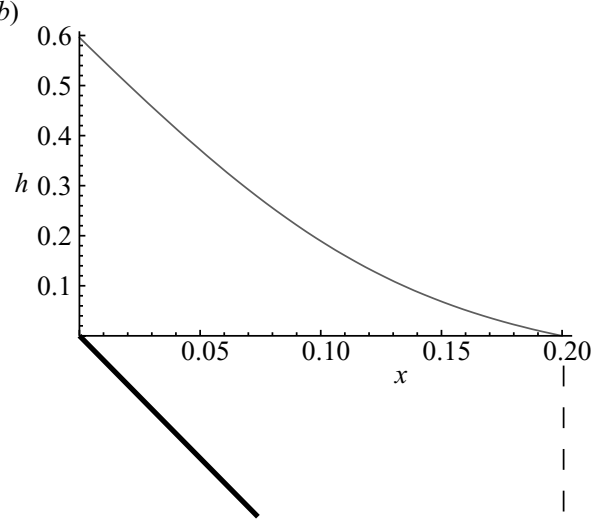

FIGURE 4. Steady scaled solutions for the viscous sheet on $(a)$ a shallow slope with $A=0.2$ and $(b)$ a steep slope with $A=5$. The value of $\epsilon$ is zero in both cases. The solid line below each graph indicates the position of the rigid slope, which continues in the $x$ direction. The position of the grounding line is shown by a vertical dashed line in each case. Note the different vertical and horizontal scales used in the two figures.

\subsection{Steady solution}

The equations and boundary conditions above admit a steady solution $\left(\dot{x}_{G}=0\right)$ in which

$$
\begin{aligned}
(h+A x)^{3} \frac{\partial h}{\partial x} & =-1, & & \\
h & =\epsilon x_{G} & & \left(x=x_{G}\right), \\
\frac{\partial h}{\partial x} & =-1 & & \left(x=x_{G}\right) .
\end{aligned}
$$

It is straightforward to combine these to determine the steady position of the grounding line

$$
x_{G}=(A+\epsilon)^{-1},
$$

where the full depth of the current $H_{G}=1$.

When $A=1$, there is a simple solution with $H \equiv 1$, so that

$$
h=1-x \text { in } 0 \leqslant x \leqslant(1+\epsilon)^{-1},
$$

For other values of $A$, the steady solution can be found straightforwardly, if laboriously, in the implicit form

$$
\begin{aligned}
a^{4}\left(x-x_{G}\right)=a( & H-1)+\frac{1}{3} \ln \left(\frac{a H-1}{a-1}\right)-\frac{1}{6} \ln \left(\frac{a^{2} H^{2}+a H+1}{a^{2}+a+1}\right) \\
- & {\left[\tan ^{-1} \frac{2}{\sqrt{3}}\left(a H+\frac{1}{2}\right)-\tan ^{-1} \frac{2}{\sqrt{3}}\left(a+\frac{1}{2}\right)\right] / \sqrt{3}, }
\end{aligned}
$$

where $a=A^{1 / 3}$.

It is perhaps more straightforward these days simply to integrate (3.7)-(3.9) numerically using standard packages. This was done to produce the graphs in figure 4 . We see that the character of the solution is different for shallow slopes $(A<1)$, shown in figure 4(a), and for steep slopes $(A>1)$, shown in figure $4(b)$. In both cases the surface of the current runs roughly parallel to the slope initially. Over steep slopes, such as those used in the experiments reported later in $\S 4$, the slope of the 
current reduces as the current nears the ocean, while the current thickens towards the grounding line. In contrast, over shallow slopes, the surface of the current dips steeply and the current thins rapidly towards the grounding line. This latter behaviour seems analogous to the situations analysed by Schoof (2007), who exploited asymptotically (as $A \rightarrow 0$ ) the fact that over shallow slopes the sheet is mostly much thicker than at the grounding line.

\subsection{Approximate solution for early time}

As an indication of the characteristic behaviour of the system at early times and to initialize our numerical solutions, given below, we analyse the case when $\epsilon=0$ in the limit $t \ll 1$. We introduce a scaled (similarity) variable $\xi=x / t^{4 / 5}$, write

$$
h=t^{1 / 5} f(\xi, t)
$$

and rewrite (3.2) as

$$
t f_{t}+\frac{1}{5} f-\frac{4}{5} \xi f^{\prime}=\left[\left(f+A \xi t^{3 / 5}\right)^{3} f^{\prime}\right]^{\prime},
$$

where subscript $t$ denotes differentiation with respect to dimensionless time and primes denote differentiation with respect to $\xi$. The boundary conditions (3.3)-(3.5) become

$$
\begin{aligned}
f^{3} f^{\prime} & =-1 \quad(\xi=0), \\
f & =0 \quad\left(\xi=\xi_{G}\right), \\
f^{\prime} & =-\frac{4}{5} \frac{t^{-6 / 5}}{A^{2} \xi_{G}} . \quad\left(\xi=\xi_{G}\right),
\end{aligned}
$$

where $\xi_{G}$ is defined by

$$
x_{G}=\xi_{G} t^{4 / 5} .
$$

Note that at early times the surface slope $-f^{\prime}$ is large, the front of the sheet is controlled kinematically and no shelf forms.

To leading order in $t \ll 1$, (3.14) with boundary conditions (3.15) and (3.16) are those describing a viscous gravity current on a horizontal plane fed by a constant volume flux (Huppert 1982), which has a similarity solution depending only on $\xi$. However, the front condition (3.17) is different from that case and requires that there be a finite slope to the gravity current at the grounding line $\xi=\xi_{G}$.

Nevertheless, the time derivative in (3.14) can be neglected to leading order and the resulting equation integrated once to give

$$
-\int_{\xi}^{\xi_{G}} f\left(\xi^{\prime}\right) \mathrm{d} \xi^{\prime}-\frac{4}{5} \xi f=\left(f+A \xi t^{3 / 5}\right)^{3} f^{\prime}+q_{G}(t),
$$

where $q_{G}(t)$ is the dimensionless volume flux at the grounding line. Given that $f=0$ at the grounding line, from (3.16), we can use this equation with (3.17) to show that

$$
q_{G}(t) \sim \frac{4}{5} A \xi_{G}^{2} t^{3 / 5} .
$$

The leading-order shape of the sheet near the grounding line at early times can be found in a form reminiscent of strained coordinates, in which the singularity of the nonlinear equation (3.14) is moved to a position $\xi=\xi_{G}+\delta(t)$. It is found by setting 


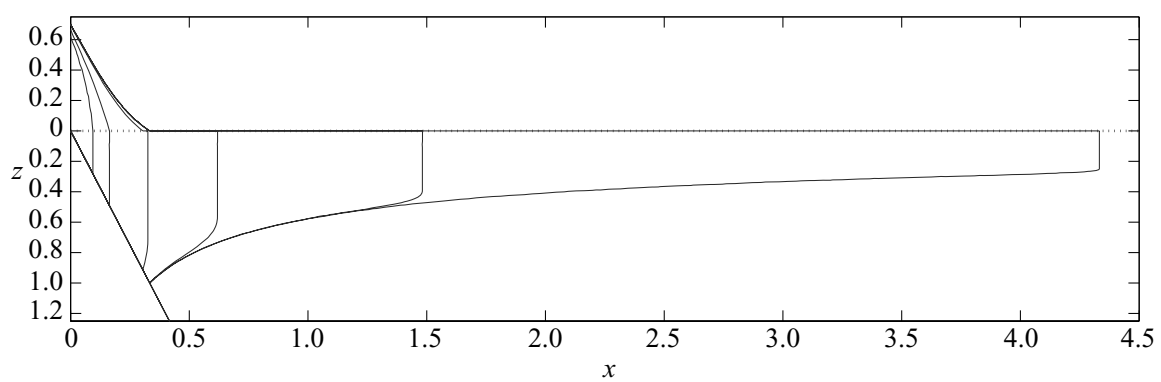

FIGURE 5. Numerically calculated evolution of the dimensionless sheet-shelf system using parameter values $A=3$ and $\epsilon=0$. From left to right, the profiles correspond to dimensionless times $0.05,0.1,0.25,0.5,1.0$ and 2.0 .

$\xi=\xi_{G}$ in the coefficients of (3.19) and integrating to obtain

$$
f \sim\left(\frac{12}{5} \xi_{G}\right)^{1 / 3}\left[\left(\xi_{G}+\delta-\xi\right)^{1 / 3}-\delta^{1 / 3}\right]
$$

where

$$
\delta \sim\left(\frac{5}{12}\right) A^{3} \xi_{G}^{2} t^{9 / 5}
$$

At a fixed small time $t>0,(3.14)$ with $f_{t}=0$ can be integrated numerically from $\xi=\xi_{G}$ backwards to $\xi=0$, starting from conditions (3.16) and (3.17) and using $\xi_{G}$ as a shooting parameter to meet the boundary condition (3.15). However, we can find $\xi_{G}$ approximately (see Huppert 1982) by applying the integral constraint on the total volume,

expressed non-dimensionally by

$$
\int_{0}^{x_{G}} h(x, t) \mathrm{d} x=q t
$$

$$
\int_{0}^{\xi_{G}} f(\xi) \mathrm{d} \xi=1,
$$

to the leading-order front solution (3.21). This gives

$$
\xi_{G} \approx\left(\frac{5}{12}\right)^{1 / 5}\left(\frac{4}{3}\right)^{3 / 5} \approx 0.997 .
$$

\subsection{Numerical solution}

As an illustration of the temporal evolution of the ice sheet and shelf, we have integrated (3.2) numerically using boundary conditions (3.3)-(3.5). This was done by mapping the domain $\left[0, x_{G}(t)\right]$ onto $[0,1]$ and using finite differences on a uniform grid in the mapped domain (see Acton, Huppert \& Worster 2001, for example), starting from the asymptotic solution (3.13) evaluated using (3.21). This determines the evolution of the sheet. Subsequently, simple quadrature was used to evaluate (2.15) for the position of Lagrangian points within the shelf, and (2.18) was used to evaluate the corresponding shelf thicknesses.

Some results are plotted in figure 5. The early transient shows the migration of the grounding line and the evolution of the profile of the sheet from the small-time asymptotic shape, which is typical of viscous gravity currents on a horizontal plane, 


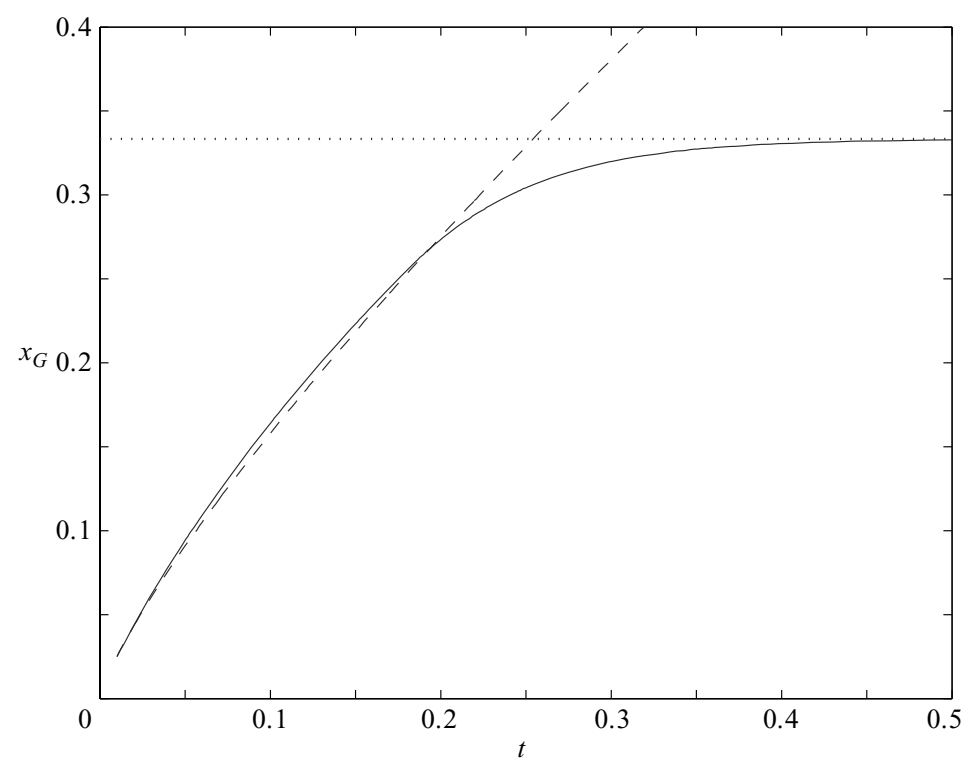

FIgURE 6. The dimensionless position of the grounding line as a function of time with parameter values $A=3$ and $\epsilon=0.1$. The solid curve shows the numerical solution, the dashed curve shows the small-time asymptotic solution, and the dotted line shows the analytically determined steady position of the grounding line.

towards the steady shape similar to that shown in figure $4(b)$. Note that there is no shelf initially. The subsequent profiles show the continuing development of the shelf after the grounding line has reached a steady position. The general shape of the shelf is similar to that observed in experiments (figure 1). This overall shape is a consequence of the history of the flux through the grounding line.

Figure 6 shows the position of the grounding line as a function of time, which evolves from the early proportionality to $t^{4 / 5}$ towards a constant value.

Some further illustrative numerical calculations are described in the discussion section below.

\section{Experimental system}

Our experiments were carried out in a Perspex tank $15 \mathrm{~cm}$ wide by $100 \mathrm{~cm}$ long by $10 \mathrm{~cm}$ deep, illustrated schematically in figure 7 . There were two distinct sets of experiments. In the first six, experiments $\mathrm{a}-\mathrm{f}$, glycerine was used to model the ice, whereas in the last four, experiments $\mathrm{A}-\mathrm{D}$, golden syrup was used. Golden syrup has a much higher viscosity than glycerine and produced thicker currents and a more two-dimensional flow.

Both glycerine and golden syrup have high densities $\left(1.26 \mathrm{~g} \mathrm{~cm}^{-3}\right.$ and $1.44 \mathrm{~g} \mathrm{~cm}^{-3}$ respectively), and a denser 'ocean' was required to ensure that the shelf floated. A saline solution using common salt $(\mathrm{NaCl})$ could not achieve this and so potassium carbonate $\left(\mathrm{K}_{2} \mathrm{CO}_{3}\right)$ solutions with densities up to $\rho_{w} \simeq 1.5 \mathrm{~g} \mathrm{~cm}^{-3}$ were used to model the sea water. The value of $g^{\prime}$ could be varied independently of the viscosity of the input fluid $v$ by changing the concentration (hence the density) of the potassium carbonate solution. The viscosity of the glycerine was altered by dilution with water. 


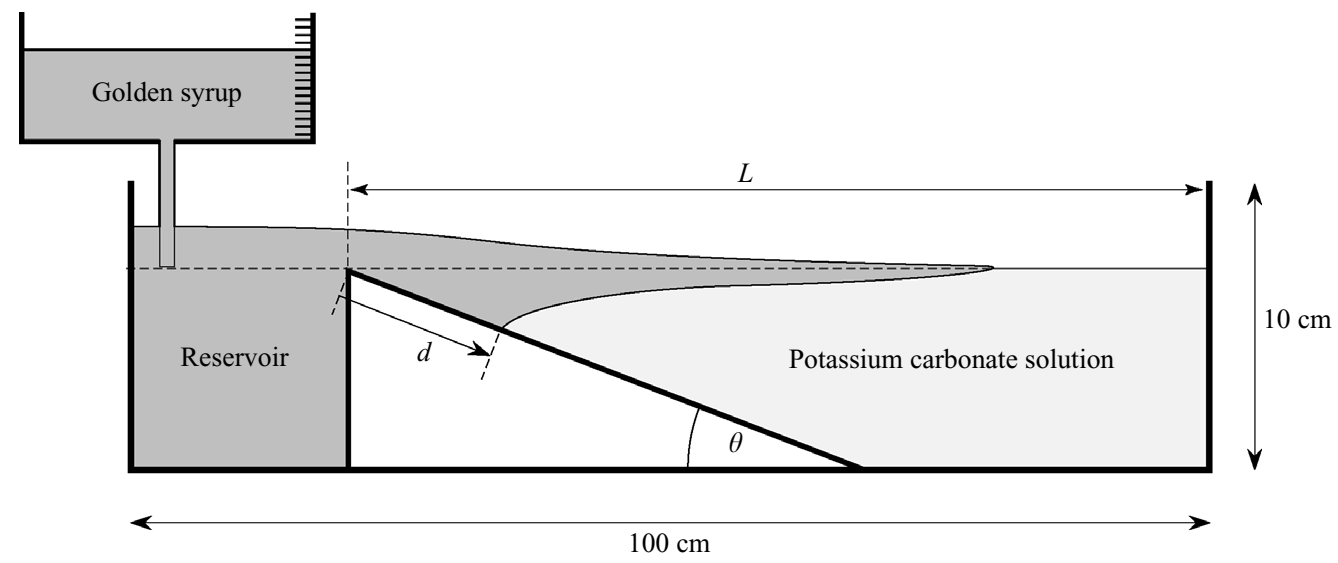

FIGURE 7. The set-up used in experiments A-D. Golden syrup is supplied at a constant rate to the reservoir at the left and spills onto a rigid slope and into a dense solution of potassium carbonate.

A Perspex ramp was secured towards one end of the tank. Its angle could be adjusted between experiments. Before each experiment, the tank was filled with aqueous $\mathrm{K}_{2} \mathrm{CO}_{3}$ solution. In the second set of experiments the distance along the ramp above the water line was always less than $2 \mathrm{~cm}$. Having only a very short length of ramp above the water line helped keep the front uniform and this keeps the system close to the ideal theoretical situation described in the previous section.

The release mechanism differed between the two series, with the second arrangement achieving a far more two-dimensional flow. For experiments a- $\mathrm{f}$ there was a small reservoir behind the ramp filled with glycerine from a cylindrical tube fitted to a measuring cylinder. In contrast, for experiments A-D we used a large reservoir between the top of the ramp and the back end of the tank, filled with golden syrup before the start of each experiment. This is the arrangement shown in figure 7. Above this reservoir a tank was suspended from which golden syrup was released at the start of an experiment through a thin slot of width $13 \mathrm{~cm}$ (i.e. just narrower than the tank) and length $1 \mathrm{~cm}$.

A constant head of glycerine or golden syrup was maintained in the supply chamber throughout each experiment to effect a constant flux. The flux rate $q_{0}$ was adjusted between experiments by altering the head height. Marks were made every $0.5 \mathrm{~cm}$ along the top of the main tank and along the ramp, and a side-view camera was used to record the experiments. The evolution of the grounding line was measured by examining the video recordings later. In each experiment, constant parameters of input flux $q_{0}$, bed slope $\alpha$, reduced gravity $g^{\prime}$, and kinematic viscosity $v$ were chosen. The values used are listed in table 1. A typical experiment is shown in movie 1.

\section{Results}

The position of the grounding line, measured as distance down the ramp, is shown as a function of time for each of the ten experiments in figure 8 . From this figure we see that the grounding line reached an almost steady position in each case apart from experiment $\mathrm{C}$, which used a small value of $\theta=5^{\circ}$, and a steady position was not reached until well after $100 \mathrm{~s}$, when the shelf had also reached the end of the tank. 


\begin{tabular}{crrlc} 
Experiment & $g^{\prime}\left(\mathrm{cm} \mathrm{s}^{-2}\right)$ & $q_{0}\left(\mathrm{~cm}^{2} \mathrm{~s}^{-1}\right)$ & \multicolumn{1}{c}{$\alpha$} & $v\left(\mathrm{~cm}^{2} \mathrm{~s}^{-1}\right)$ \\
a & 62.8 & 0.105 & 0.25 & 8.3 \\
$\mathrm{~b}$ & 156.9 & 0.210 & 0.25 & 8.3 \\
$\mathrm{c}$ & 179.5 & 0.420 & 0.25 & 8.3 \\
$\mathrm{~d}$ & 179.5 & 0.420 & 0.0875 & 8.3 \\
$\mathrm{e}$ & 179.5 & 0.420 & 0.25 & 1.0 \\
$\mathrm{f}$ & 32.4 & 0.105 & 0.25 & 9.4 \\
$\mathrm{~A}$ & 32.9 & 0.464 & 0.27 & 380 \\
$\mathrm{~B}$ & 32.9 & 0.464 & 0.27 & 380 \\
$\mathrm{C}$ & 32.9 & 0.464 & 0.0105 & 380 \\
$\mathrm{D}$ & 32.9 & 0.464 & 0.78 & 380
\end{tabular}

TABLE 1. Values used for the input parameters in each experiment.

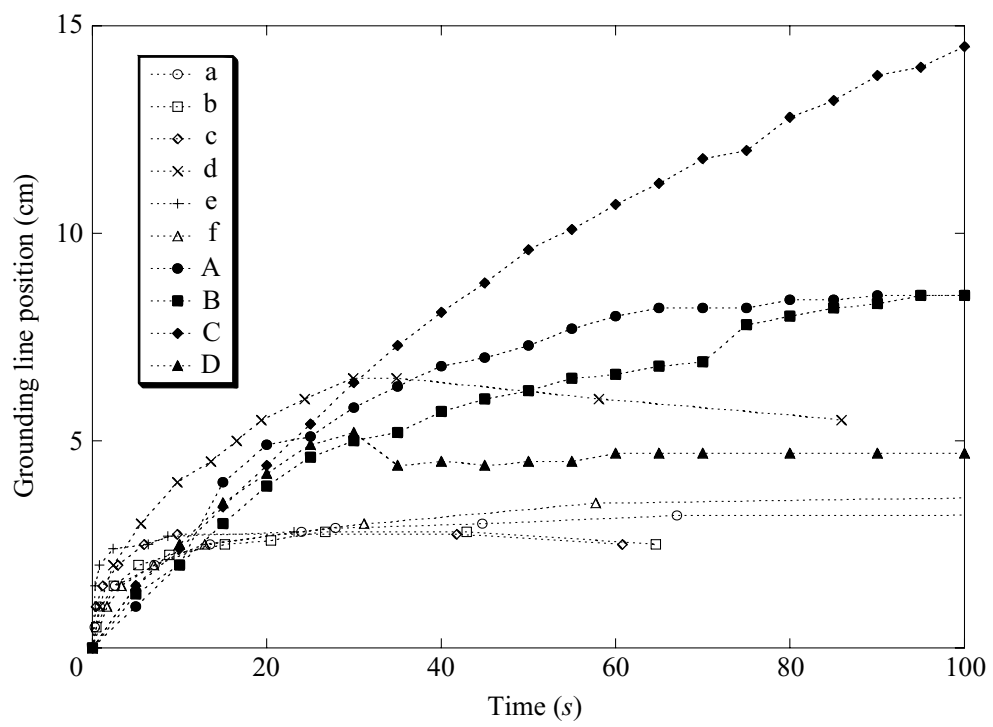

FIGURE 8. The downslope position of the grounding line, indicated by $d$ in figure 7 , as a function of time for each of the experiments.

These measurements are only accurate to roughly half a centimetre, partly because of variations across the width of the tank and partly because it is sometimes unclear from the observations whether the viscous fluid is over-riding the fluid it is displacing or whether it is actually detaching to form a shelf. There were indications in some experiments (most noticeably experiment D) that the current 'lifted off' at some point, perhaps associated with the transition between kinematic and dynamic control of the front of the sheet described between (2.34) and (2.35), and that the grounding line then appeared to recede slightly.

Our theoretical prediction for the steady position of the grounding line, given in dimensionless form as $(A+\epsilon)^{-1}$ in $(3.10)$, is

$$
x_{G}=\frac{1}{\alpha} \frac{\rho}{\rho_{w}}\left(\frac{g}{g^{\prime}}\right)^{1 / 6}\left(\frac{6 v q_{0}}{g}\right)^{1 / 3} .
$$




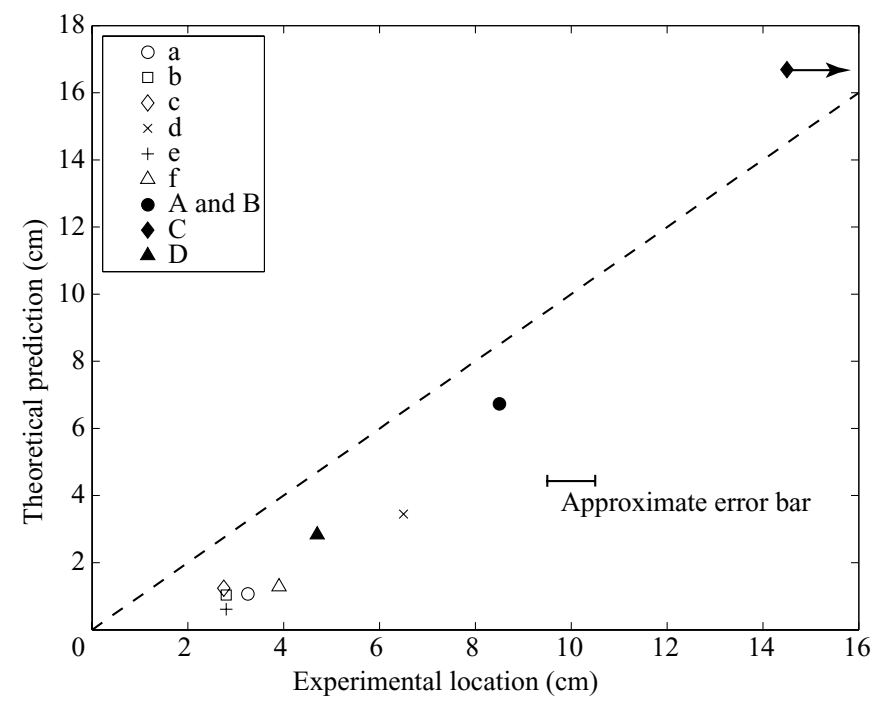

FIGURE 9. A comparison between the maximum location of the grounding line measured in our experiments and our theoretical predictions. The arrow on the data point for experiment $\mathrm{C}$ indicates that the grounding line in this experiment was still advancing.

A comparison between this prediction and our experimental results is shown in figure 9 . We see that though the grounding line reached a position broadly consistent with our theoretical predictions, it consistently settled some $2 \mathrm{~cm}$ further along the slope than predicted, apart from in experiment $\mathrm{C}$, in which the grounding line may not have reached its steady position before the experiment ceased. Note that there are no adjustable parameters in the theoretical prediction, which is clearly giving the right magnitude of variation of grounding-line position as the external parameters varied by more than two orders of magnitude. Some reasons for the discrepancy between theory and experiment are discussed in the following section.

\section{Discussion}

There are two significant ways in which our experiments differ from the ideal situation we have analysed theoretically. Firstly, as syrup is introduced it displaces some of the water, and the water line (sea level) rises. Secondly, the theoretical model is purely two-dimensional, whereas the shelf in the experiments is influenced by viscous shear stresses exerted by the sidewalls of the tank. We have used our numerical model to assess these effects as follows.

It is straightforward to account for the rise in sea level by writing

$$
b(x, t)=\alpha x+\left[q_{0} t-\int_{0}^{x_{N}(t)} h(x, t) \mathrm{d} x\right] / L,
$$

where $L$ is the horizontal distance from the top of the ramp to the other end of the tank from the reservoir. This expression can be differentiated with respect to time and rearranged to show that

$$
\frac{\partial b}{\partial t}=\frac{\rho}{\rho_{w}} \frac{q_{G}(t)}{L-x_{G}(t)}
$$




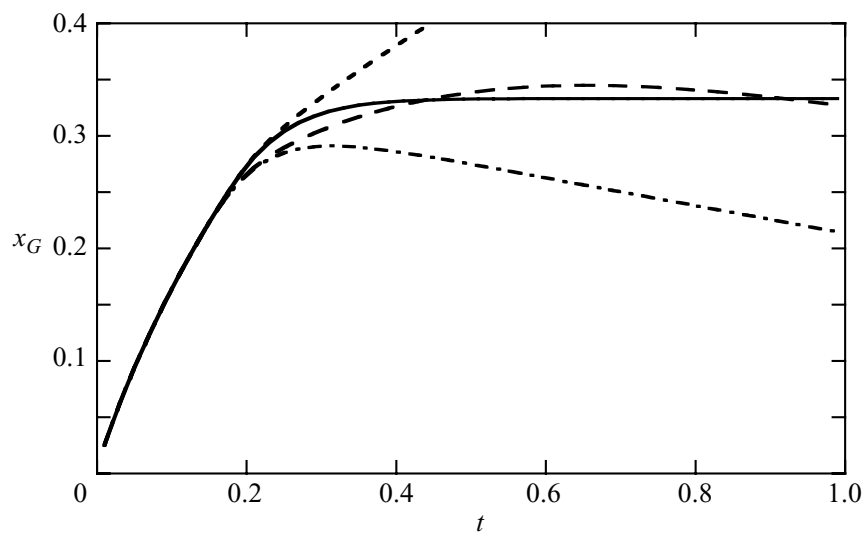

FIGURE 10. Simulations incorporating the effects of sea-level rise and sidewall stresses in a finite tank. For reference, the solid curve reproduces the ideal calculation from figure 5 . The dot-dash curve shows the results of including sea-level rise, while the short-dashed curve shows the effect of sidewall stress with no sea level rise, calculated with $k=50$. The long-dashed curve shows the combined effects of sea-level rise and sidewall stress with $k=50$. The other parameters are the same as those used in figure 5, with dimensionless values of $L=4.5$ and $W=0.75$.

so that

$$
b(x, t)=\alpha x+\frac{\rho}{\rho_{w}} \int_{0}^{t} \frac{q_{G}(\tau)}{L-x_{G}(\tau)} \mathrm{d} \tau .
$$

These expressions are used to modify (2.25), (2.26) and (2.33), which we integrated in dimensionless form to produce the dot-dash curve in figure 10 . We see that the rise in sea level causes the grounding line eventually to recede and to fall short of the previously calculated steady position.

A proper treatment of the effects of the sidewalls of the tank would involve an analysis of the three-dimensional behaviour of the shelf. However, the overall effect is to provide an additional normal stress to the sheet at the grounding line, which we model here by replacing $(2.7)$ with

$$
N=-\frac{1}{2} \rho g H^{2}+4 \mu H \frac{\partial u}{\partial x}-k \mu \frac{u_{G}}{W} \frac{A_{\text {shelf }}}{W},
$$

where $u_{G}=q_{G} / H_{G}$ is the mean velocity of the fluid in the shelf at the grounding line,

$$
A_{\text {shelf }}=\int_{x_{G}}^{x_{N}} H(x, t) \mathrm{d} x
$$

is the cross-sectional area of the shelf in contact with the sidewalls of the tank, $W$ is the width of the tank and $k$ is a dimensionless constant. In other words, we add a stress proportional to a characteristic horizontal shear in the shelf and to the area of viscous contact.

The effect of the additional stress from the sidewalls, without the effect of sea-level rise, is to allow the grounding line to advance indefinitely, so long as the sidewalls themselves extend indefinitely. However, as shown in figure 10, the combination of the additional stress and the rising sea level in a finite tank is to cause a levelling out and eventual recession of the grounding line, in accord with our experimental observations. These calculations indicate that the discrepancies between theory and experiment shown in figure 9 may well be accountable by the effects we have just described, though clearly more work is needed to confirm this. 


\section{Conclusions}

We have shown that important characteristics of the dynamics of marine ice sheets can be modelled using fluid-mechanical laboratory experiments involving simple fluids. Our preliminary experiments show the migration of the grounding line between a grounded sheet and freely floating shelf towards a steady position, confirming the stability of the grounding line on a down slope both with respect to lateral variations and to its overall position.

We have developed and solved a simple model of a Newtonian viscous sheet-shelf system. A particular feature of our model is an explicit evolution equation for the position of the grounding line, which allows the temporal evolution of the whole system to be calculated straightforwardly as a free-boundary problem. This approach has similarities to the Stefan problems that have been well studied in the context of solidification, for example.

Comparisons between our theoretical predictions and experimental results indicate that our model embodies the correct dependences on the physical parameters of the system but highlight the roles played in the experiments by the rise in sea level caused by the addition of fluid to the tank and by horizontal viscous stresses acting on the shelf exerted by the sidewalls. These important aspects are the subject of our ongoing research.

We would like to thank Dr Mark Hallworth for valuable help with experiments and to Richard Katz and Luke Rajah for providing independent checks on some of our computations. The research of HEH is partially supported by a Royal Society Wolfson Research Merit Award. He is also grateful to the Chancellor of the University of New South Wales, David Gonski, for allowing him to work at this desk while considering revisions of the paper.

Supplementary movies are available at journals.cambridge.org/flm.

\section{REFERENCES}

Acton, J. M., Huppert, H. E. \& Worster, M. G. 2001 Two-dimensional viscous gravity currents flowing over a deep porous medium. J. Fluid Mech. 440, 359-380.

HuPPERT, H. E. 1982 The propagation of two-dimensional and axisymmetric viscous gravity currents over a rigid horizontal surface. J. Fluid Mech. 121, 43-58.

Huppert, H. E. 2000 Geological fluid mechanics. In Perspectives in Fluid Dynamics: A Collective Introduction to Current Research (ed. G. K. Batchelor, H. K. Moffatt \& M. G. Worster), pp. 447-506. Cambridge University Press.

IPCC REPORT 2007 Intergovernmental Panel on Climate Change Fourth Assessment Report.

Nowicki, S. M. J. \& Wingham, D. J. 2008 Conditions for a steady ice sheet-ice shelf junction. Earth Planet Sc. Lett. 265, 246-255.

Paterson, W. S. B. 1994 The Physics of Glaciers, 3rd edn. Pergamon.

Schoof, C. 2007 Marine ice sheet dynamics. Part I: the case of rapid sliding. J. Fluid Mech. 573, $27-55$.

Vieli, A. \& PaYne, A. J. 2005 Assessing the ability of numerical ice sheet models to simulate grounding line migration. J. Geophys. Res.-Earth 110, F01003.

WeErTMAn, J. 1974 Stability of the junction of an ice sheet and an ice shelf. J. Glac. 13 (67), 3-11.

WiLCHINSKY, A. V. 2001 Studying ice sheet stability using the method of separation of variables. Geophys. Astrophys. Fluid Dyn. 94, 15-45.

Wilchinsky, A. V. \& Chugunov, V. A. 2000 Ice stream-ice shelf transition: theoretical analysis of two-dimensional flow. Ann. Glaciol. 30, 153-162. 